\title{
Insufficient evidence of effect of periodontal treatment on prevention or management of cardiovascular disease
}

\author{
Abstracted from \\ Li C, Lv Z, Shi Z, Zhu Y, Wu Y, Li L, Iheozor-Ejiofor Z. \\ Periodontal therapy for the management of cardiovascular disease in patients with chronic periodontitis. \\ Cochrane Database Syst Rev 2014; 8: Art. No. CD009197. DOI: 10.1002/14651858.CD009197.pub2. \\ Address for correspondence: Luisa Fernandez Mauleffinch, Review Group Co-ordinator, \\ Cochrane Oral Health Group, School of Dentistry, The University of Manchester, Coupland III Building, \\ Oxford Road, Manchester, M13 9PL, UK. E-mail: luisa.fernandez@manchester.ac.uk
}

\section{Question: Does treating periodontal disease in patients with chronic periodontal disease help prevent or manage cardiovascular disease (CVD)?}

Data sources The Cochrane Oral Health Group's Trials Register, the Cochrane Central Register of Controlled Trials (CENTRAL), Medline, Embase, CINAHL, OpenGrey, the Chinese BioMedical Literature Database, the China National Knowledge Infrastructure and the VIP database, the US National Institutes of Health Trials Register, the World Health Organisation (WHO) Clinical Trials Registry Platform and Sciencepaper Online databases were searched with no restrictions on language or date.

Study selection Two reviewers independently selected studies and only randomised controlled trials (RCTs) and quasi-RCTs with follow-up times $\geq$ one year were considered.

Data extraction and synthesis Data extraction and risk of bias assessment were carried out independently and in duplicate. Standard Cochrane methodological approaches were used.

Results Only one trial involving 303 patients that compared scaling and root andomiz (SRP) with community care was included. It was considered to be at high risk of bias. No data on deaths (all-cause or CVD-related) were reported. There was insufficient evidence to determine the effect of SRP and community care in reducing the risk of CVD recurrence in patients with chronic periodontitis (risk ratio (RR) 0.72 ; $95 \%$ confidence interval $(\mathrm{Cl}) 0.23$ to 2.22 ; very low quality evidence). The effects of SRP compared with community care on highsensitivity C-reactive protein (hs-CRP) (mean difference (MD) 0.62; -1.45 to 2.69 ), the number of patients with high hs-CRP (RR 0.77; $95 \% \mathrm{Cl} 0.32$ to 1.85 ) and adverse events (RR 9.06; $95 \% \mathrm{Cl} 0.49$ to $166.82)$ were also not statistically significant. The study did not assess modifiable cardiovascular risk factors, other blood test results, heart function parameters or revascularisation procedures.

Conclusions We found very low quality evidence that was insufficient to support or refute whether periodontal therapy can prevent the recurrence of CVD in the long term in patients with chronic periodontitis. No evidence on primary prevention was found.

This paper is based on a Cochrane Review published in the Cochrane Library 2014, issue 8 (see www.thecochranelibrary.com for information). Cochrane Reviews are regularly updated as new evidence emerges and in response to feedback, and the Cochrane Library should be consulted for the most recent version of the review.

\section{Commentary}

The objective of this review is to investigate the effects of periodontal therapy to prevent the occurrence of, and to manage the recurrence of cardiovascular disease (CVD) in patients with chronic periodontitis.

The outcomes selected by the authors for the clinical question were: CVD related death and CVD signs such as angina, myocardial infarction and cerebrovascular accidents as the main outcomes and secondary outcomes were represented by risk factors, biomarkers and surrogate end points for cardiovascular disease.

The comprehensive methodology used to conduct the review included an extensive search of andomized clinical trials in no other than English and Chinese databases and journals. The final selection of articles included one study.

The selected study critically appraised in the review provides information of low quality evidence to support or to refute periodontal treatment to prevent or to manage the occurrence or recurrence of cardiovascular disease in patients with chronic periodontitis. The study analysed the number of cardiovascular events and level of C-reactive protein (CRP) in patients with periodontal disease. As a note, CRP is a marker for inflammation. However, it is not a specific marker for periodontal disease. Data for both outcomes were not statistically significant.

As the authors mentioned, it is important that future studies should consider certain cofounders that could impact periodontal disease and cardiovascular disease, such as acute inflammation and diabetes.

Additionally, age, congenital versus acquired CVD and diet could be important and worthy of inclusion, since diet could have an impact on systemic inflammation.

If future trials can shed more light on how important the treatment of periodontal disease is in the prevention and recurrence of cardiovascular disease, the results could give us an important piece of information to instruct patients on the significance of regular care and understand the impact of the control of periodontal disease.

There is no doubt that the link between cardiovascular disease and chronic periodontitis is one that encourages controversy and debate.

In 2012 an extensive review produced by the American Heart Association Scientific Affairs section with the support of the American Dental Association Council of Scientific Affairs and endorsed by the World Heart Federation concluded that observational studies support an association between periodontal disease (PD) and atherosclerotic vascular disease (ASVD) independent of 
known cofounders. They do not, however, support a causative relationship. Although periodontal interventions result in a reduction in systemic inflammation and endothelial dysfunction in shortterm studies, there is no evidence that they prevent ASVD or modify its outcomes. ${ }^{1}$

Despite the results of the review, whether or not or how significant an association there is between prevention and or control of systemic diseases, it is important to promote self-care to all of our patients and to provide them adequate treatment and prevention for all the oral health diseases.
Marc Henschel and Analia Veitz Keenan

NYU College of Dentistry, New York, USA

1. Lockhart PB, Bolger AF, Papapanou PN, et al, on behalf of the American Heart Association Rheumatic Fever, Endocarditis, and Kawasaki Disease Committee of the Council on Cardiovascular Disease in the Young, Council on Epidemiology and Prevention, Council on Peripheral Vascular Disease, and Council on Clinical Cardiology. Periodontal disease and atherosclerotic vascular disease: does the evidence support an independent association?: a scientific statement from the American Heart Association. Circulation 2012; 125: 2520-2544.

Evidence-Based Dentistry (2015) 16, 17-18. doi:10.1038/sj.ebd.6401079 